\title{
ALGORITHM OF $J$-FACTORIZATION OF RATIONAL MATRICES WITH ZEROS AND POLES ON THE IMAGINARY AXIS
}

\author{
VLADIMIR B. LARIN
}

Received 27 October 2002

\begin{abstract}
The problem of $J$-factorization of rational matrices, which have zeros and poles on the imaginary axis, is reduced to construction of the solutions of two algebraic Riccati equations. For construction of these solutions, it is offered to use appropriate algorithms. These algorithms permit to find the solutions in cases when the Hamiltonian matrices, which are corresponding to these equations, have eigenvalues on the imaginary axis. Algorithms of factorization, which had been offered, permit to find the solution of the problem when the matrix, which will be factored, has zeros at infinity.
\end{abstract}

2000 Mathematics Subject Classification: 93B36, 93B40, 15A23.

1. Introduction. It is known that the procedure of $J$-factorization of rational matrices arises in the modern control theory [6]. For the development of the numerical algorithms of realization of this procedure, the investigations of the connection between the procedure of $J$-factorization and the solution of algebraic Riccati equation (ARE) [8] are very important. The point is that procedure of $J$-factorization in some cases can be reduced to the solution of ARE [10]. However, if the matrix has zeros and poles on the imaginary axis, it should be taken into account at reduction of this procedure to solution of ARE. Thus in this case, it is necessary to remember that for solution of ARE it is necessary to use the special algorithms (the Hamiltonian matrix, corresponding to ARE, has eigenvalues on the imaginary axis). Below, the algorithm of $J$-factorization of the rational matrices with zeros and poles on the imaginary axis is stated. This algorithm has been published in [15].

Before the formulation of the problem, we will give some known facts. According to [2, 3], the problem of factorization of a rational matrix relatively to the imaginary axis is formulated as follows. The Hermitian positive definite on the imaginary axis rational matrix $\Phi(s)$ is given, that is,

$$
\Phi(s)=\Phi_{*}(s), \quad \Phi_{*}(s)=\Phi^{T}(-s), \quad \Phi(i \omega)>0 \text { for any real } \omega .
$$

The superscript $T$ denotes transposition. It is necessary to determine the matrix $G(s)$, which satisfies the relation

$$
\Phi(s)=G_{*}(s) G(s),
$$


and the matrices $G(s)$ and $G^{-1}(s)$ which have no poles in the open right halfplane $\mathbb{C}_{+}\{s$, Res $>0\}$. Frequently, in such problems the matrix $\Phi(s)$ is stated as in [4]:

$$
\Phi(s)=R+B^{T}(I s-A)_{*}^{-1} C+C^{T}(I s-A)^{-1} B+B^{T}(I s-A)_{*}^{-1} Q(I s-A)^{-1} B .
$$

The identity matrices with the corresponding dimensions are denoted by $I$. If the eigenvalues of the matrix $A$ do not belong to $\mathbb{C}_{+}$and there exists $R^{-1}(\Phi(\infty)$ is invertible), the problem of determination of $G(s)$ can be reduced to solution of ARE. So, according to [4],

$$
\begin{gathered}
G(s)=L+K^{T}(I s-A)^{-1} B, \\
R=L^{T} L, \quad K^{T}=L^{-T}(P B+C)^{T}, \quad L^{-T}=\left(L^{-1}\right)^{T} .
\end{gathered}
$$

In this relation, the matrix $P$ is the stabilizing solution of ARE

$$
P A+A^{T} P+Q-K K^{T}=0
$$

that is the solution which ensures disposition of the eigenvalues of the matrix $A_{C}=A-B R^{-1}(P B+C)^{T}$ in the left half-plane. In this case the matrix

$$
G^{-1}(s)=L^{-1}-L^{-1} K^{T}\left(I s-A_{c}\right)^{-1} B L^{-1}
$$

has no poles in $\mathbb{C}_{+}$. However the poles of $G(s)$ are determined by eigenvalues of the matrix $A$. For generalization of this problem in the case when the matrix (1.3) has zeros on the imaginary axis and $R \geq 0$, see [5].

The problem of $J$-factorization of the matrix (1.3) was considered in [10]. So, if there exists $R^{-1}$, the problem of $J$-factorization of the matrix (1.3) consists in representing it as follows (see [10, relation (89)]):

$$
\begin{gathered}
\Phi(s)=G_{*}(s) R G(s), \\
G(s)=I+\tilde{K}^{T}(I s-A)^{-1} B, \\
\tilde{K}=(P B+C) R^{-1}, \quad G^{-1}(s)=I-\tilde{K}^{T}\left(I s-A_{C}\right)^{-1} B, \quad A_{C}=A-B R^{-1}(P B+C)^{T} .
\end{gathered}
$$

The matrix $P$ is the stabilizing solution of the ARE

$$
P A+A^{T} P-\tilde{K} R \tilde{K}^{T}+Q=0 .
$$

It is possible to note that if zeros of $\Phi(s)$ lay on the imaginary axis, then the Hamiltonian matrix, which corresponds to ARE (1.10), will also have eigenvalues on the imaginary axis. In this case, it will be difficult to use the standard procedures for solution of ARE (Schur method [16], the matrix sign function method [9]) for determination of the stabilizing solution of ARE (1.10). In this case, we can use algorithms [7, 12, 13, 14] for solution of ARE. However, it is 
necessary to note that, if we use such algorithm of factorization, the poles of $G(s)$, as well as in (1.4), are determined by eigenvalues of the matrix $A$.

There is the problem of generalization of the algorithm [10] of $J$-factorization of the matrix (1.3) (representing this matrix as (1.7)). This algorithm should guarantee lack of zeros and poles of the matrix $G(s)$ in $\mathbb{C}_{+}$at an arbitrary spectrum of the matrix $A$.

In $[1,11]$, algorithm of factorization of the matrix (1.3), which guaranteed a lack of zeros and poles of the matrix $G(s)$ in $\mathbb{C}_{+}$, was offered. Algorithm has permitted to solve the problem with singular matrix $R$. However, it was based on the assumption that the matrix $A$ has no eigenvalues on the imaginary axis.

It is possible to construct algorithm of $J$-factorization of the matrix (1.3), which has zeros and poles on the imaginary axis, by generalizing algorithms of factorization $[11,14]$ and using for solution of ARE the algorithms in [12, 13, 14] (see Appendix A). This algorithm allows to solve the problem with singular matrix $R$. In contrast to the algorithm in $[12,13]$, in this algorithm, the assumption that the matrix $A$ has no eigenvalues on the imaginary axis is not used.

2. Existence of $R^{-1}$. As it was noted, if the matrix $A$ has eigenvalues in $\mathbb{C}_{+}$, the algorithm of $J$-factorization, defined by relations (1.7), (1.10), does not ensure a lack of poles of the matrix $G(s)$ in $\mathbb{C}_{+}$. Therefore, if the matrix $A$ has eigenvalues in $\mathbb{C}_{+}$, it is necessary to modify this algorithm. This modification is connected with an additional procedure of $J$-factorizations of some auxiliary matrix. In this connection, we will deduce the expression for a matrix $\Phi^{-T}(s)\left(\Phi^{-T}(s)=\left(\Phi^{-1}(s)\right)^{T}\right)$ using the relation (1.9):

$$
\begin{aligned}
\Phi^{-T}(s)= & G_{*}^{-T} R^{-1} G^{-T}(s) \\
= & R^{-1}-\tilde{K}^{T}\left(I s-A_{c}^{T}\right)_{*}^{-1} B R^{-1}-R^{-1} B^{T}\left(I s-A_{c}^{T}\right)^{-1} \tilde{K} \\
& +\tilde{K}^{T}\left(I s-A_{c}^{T}\right)_{*}^{-1} B R^{-1} B^{T}\left(I s-A_{c}^{T}\right)^{-1} \tilde{K} .
\end{aligned}
$$

Note that the matrix $A_{c}$ has no eigenvalues in $\mathbb{C}_{+}$. In an outcome of $J$-factorization, similar to (1.7), (1.10), we will present the matrix $\Phi^{-T}(s)$ as follows:

$$
\begin{gathered}
\Phi^{-T}(s)=G_{0 *}(s) R^{-1} G_{0}(s), \\
G_{0}(s)=I+\tilde{K}_{1}^{T}\left(I s-A_{c}^{T}\right)^{-1} \tilde{K}, \quad \tilde{K}_{1}=\left(S \tilde{K}-B R^{-1}\right) R .
\end{gathered}
$$

The matrix $S$ is a stabilizing solution of the following ARE:

$$
S A^{T}+A S-S \tilde{K} R \tilde{K}^{T} S=0,
$$

that is a solution by which the matrix $A^{T}-\tilde{K} R \tilde{K}^{T} S$ has no eigenvalues in $\mathbb{C}_{+}$.

We will note that matrices $G_{0}(s)$ and

$$
G_{0}^{-1}(s)=I-\tilde{K}_{1}^{T}\left(I s-A_{c c}\right)^{-1} \tilde{K},
$$


have no poles in $\mathbb{C}_{+}$because the matrix

$$
A_{\mathcal{C C}}=A^{T}-\tilde{K} R \tilde{K}^{T} S
$$

has no eigenvalues in $\mathbb{C}_{+}$. It is essential that its spectrum is determined only by the spectrum of the matrix $A$. Really, the Hamiltonian matrix, which is corresponding to the ARE (2.3), has the form

$$
H=\left[\begin{array}{cc}
A^{T} & -\tilde{K} R \tilde{K}^{T} \\
0 & -A
\end{array}\right] .
$$

Let $\Lambda_{-}$be eigenvalues of the matrix $A$, at which $\operatorname{Re}(\lambda)<0$ and, accordingly, $\Lambda_{0}(\operatorname{Re}(\lambda)=0), \Lambda_{+}(\operatorname{Re}(\lambda)>0)$. Eigenvalues of the matrix $H$ will be accordingly $\pm \Lambda_{-}, \pm \Lambda_{0}$, and $\pm \Lambda_{+}$. Therefore, the spectrum of a matrix $A_{c c}$ will be a union of $\Lambda_{-}, \Lambda_{0}$, and $-\Lambda_{+}$.

Thus, relation (2.2) allows to modify algorithm of factorization (1.7) as follows:

$$
\begin{gathered}
\Phi(s)=\tilde{G}_{*}(s) R \tilde{G}(s), \\
\tilde{G}(s)=\tilde{G}_{0}^{-T}(s)=I-\tilde{K}^{T}\left(I s-A+S \tilde{K} R \tilde{K}^{T}\right)^{-1}(S \tilde{K} R-B), \\
\tilde{G}^{-1}(s)=I+\tilde{K}^{T}\left(I s-A+B R^{-1}(P B+C)^{T}\right)^{-1}(S \tilde{K} R-B), \quad \tilde{K}=(P B+C) R^{-1} .
\end{gathered}
$$

The matrices $P$ and $S$ are stabilizing solutions of AREs (1.10) and (2.3), respectively.

3. Computation of the matrix $S$. In a common case (when the matrix (1.3) has zeros on the imaginary axis) for determination of the matrices $P, S$, it is possible to use algorithms in [7, 12, 13, 14]. However, for construction of a solution of ARE (2.3) (determination of the matrix $S$ ), it is possible to reduce to a determination of a solution of the Lyapunov equation. So, let the orthogonal matrix $U$ reduces the matrix $A$ to the upper triangular Schur form

$$
\bar{A}=U A U^{T}=\left[\begin{array}{cc}
A_{+} & A_{0} \\
0 & A_{-}
\end{array}\right],
$$

where $\operatorname{Re} \lambda\left(A_{+}\right)>0, \operatorname{Re} \lambda\left(A_{-}\right) \leq 0$. For a determination of a matrix $U$, it is possible to use procedures schur.m and schord.m of the Matlab package. We will transform ARE (2.3) by using the matrix $U$ :

$$
\begin{gathered}
\bar{S} \bar{A}^{T}+\bar{A} \bar{S}-\bar{S} \bar{G} \bar{S}=0, \\
\bar{G}=U \tilde{K} R \tilde{K}^{T} U^{T}=\left[\begin{array}{ll}
g_{1} & g_{2} \\
g_{2}^{T} & g_{3}
\end{array}\right], \quad \bar{S}=U S U^{T} .
\end{gathered}
$$


The sizes of blocks $g_{i}(i=1,2,3)$ correspond to the partitioning (3.1). We will search for the solution of ARE (2.3) as

$$
\bar{S}=\left[\begin{array}{cc}
S_{+} & 0 \\
0 & 0
\end{array}\right] .
$$

The sizes of blocks $S_{+}$in (3.3) and $A_{+}$in (3.1) coincide.

As follows from (3.1) and (3.3), the matrix $S_{+}$satisfies the following ARE:

$$
S_{+} A_{+}^{T}+A_{+} S_{+}-S_{+} g_{1} S_{+}=0 .
$$

We will assume that ARE (3.4) has a stabilizing solution $S_{+}$and the matrix $S_{+}$ is invertible. In this case, the matrix $X=S_{+}^{-1}$ is uniquely determined by the Lyapunov equation

$$
A_{+}^{T} X+X A_{+}-g_{1}=0
$$

Thus, the desired solution of ARE (2.3) has the form

$$
S=U^{T}\left[\begin{array}{cc}
X^{-1} & 0 \\
0 & 0
\end{array}\right] U,
$$

where the matrix $X$ is determined by the Lyapunov equation (3.5).

Note that if the matrix $A$ has no eigenvalues in $\mathbb{C}_{+}$, the stabilizing solution of ARE (2.3) is $S=0$. In this case, the matrices $\tilde{G}(s)$ and $\tilde{G}^{-1}(s)$, which are appearing in (2.7), coincide with $G(s)$ and $G^{-1}(s)$, which are determined by expressions (1.8) and (1.9).

4. Case $R=0$. Let in (1.3) $R=0$. As in $[1,11]$, it is possible to modify problem of $J$-factorization of matrix (1.3) as follows. After multiplying the matrix $\Phi(s)$ on the left-hand side by the matrix $\Psi_{*}(s)$ and on the right-hand side by $\Psi(s)$ (the matrices $\Psi(s)$ and $\Psi^{-1}(s)$ have no poles in $\mathbb{C}_{+}$), we obtain

$$
\begin{gathered}
\Phi_{1}(s)=\Psi_{*}(s) \Phi(s) \Psi(s)=G_{1 *}(s) R_{1} G_{1}(s), \\
G_{1}(s)=G(s) \Psi(s) .
\end{gathered}
$$

We note that the zeros and poles of the matrix $\Psi(s)$ should not coincide with the zeros and poles of the matrix $\Phi(s)$, that is, in the process of evaluation of the matrix $\Phi_{1}(s)$ cancellation of the zeros and the poles should not happen. After the matrix $\Phi_{1}(s)$ has been factorized (the matrix $G_{1}(s)$ is found), desired solution (matrix $G(s)$ ) is determined by the following relation:

$$
G(s)=G_{1}(s) \Psi^{-1}(s) .
$$

It is expedient to select the matrix $\Psi(s)$ in such a manner that the structure of the matrix $\Phi_{1}(s)$ would be similar to the matrix (1.3), but $\Phi_{1}(\infty)$ would be 
invertible. Being guided by these reasons, we select as the matrix $\Psi(s)$ the following matrix:

$$
\Psi(s)=I s+\alpha I
$$

where the constant $\alpha>0$ does not belong to the spectrum of matrices $A$ and $-A$. This matrix satisfies the above-noted conditions of a lack of poles of matrices $\Psi(s), \Psi^{-1}(s)$ in $\mathbb{C}_{+}$. Besides, it is impossible to cancel the poles of $\Phi(s)$ and zeros of $\Psi(s), \Psi_{*}(s)$.

Further, for simplification of the calculations, we consider $\alpha=1$. We note that this restriction $( \pm 1$ does not belong to a spectrum of a matrix $A$ ) is not essential. Really, substituting $s$ by $\alpha \bar{s}(\alpha>0)$, it is possible to transform the matrix $\Phi(s)$ in such a manner that the matrix $\Phi(\bar{s})$ has no poles equal to \pm 1 [1] (see Example 4.3). Taking into consideration that

$$
B(I s+I)=(I s+I) B
$$

for appropriate sizes of the identity matrices, which appear in the left- and right-hand sides of (4.4), and also

$$
(I s-A)^{-1}(I s+I)=(I s-A)^{-1}(A+I)+I,
$$

we have, for $C^{T} B=B^{T} C$,

$$
\begin{aligned}
& \Phi_{1}(s)=\Psi_{*}(s) \Phi(s) \Psi(s)= R_{1}+B_{1}^{T}(I s-A)_{*}^{-1} C_{1}+C_{1}^{T}(I s-A)^{-1} B_{1} \\
&+B_{1}^{T}\left(I s-A_{*}\right)^{-1} Q(I s-A)^{-1} B_{1}, \\
& B_{1}=(A+I) B, C_{1}=(I-A) C+Q B, R_{1}=B^{T} Q B-C^{T} A B-B^{T} A^{T} C .
\end{aligned}
$$

Thus, in the case of existence of $R_{1}^{-1}$ and $C^{T} B=B^{T} C$, for determination of the matrix $G_{1}(s)$, it is possible to use the above-circumscribed algorithm of $J$-factorization.

So, according to (2.7),

$$
\begin{aligned}
& G_{1}(s)=I-\tilde{K}^{T}(I S-\tilde{A})^{-1}\left(S \tilde{K} R_{1}-B_{1}\right), \\
& \tilde{A}=A-S \tilde{K} R_{1} \tilde{K}^{T}, \quad \tilde{K}=\left(P B_{1}+C_{1}\right) R_{1}^{-1}
\end{aligned}
$$

the matrices $P$ and $S$ are stabilizing solutions of the following ARE:

$$
\begin{gathered}
P A+A^{T} P-\tilde{K} R_{1} \tilde{K}^{T}+Q=0, \\
S A^{T}+A S-S \tilde{K} R_{1} \tilde{K}^{T} S=0 .
\end{gathered}
$$

We will pass to determination of the matrix $G(s)$, which is defined by (4.2). According to assumption, the numbers \pm 1 do not belong to the spectrum of the matrix $A$. Taking into consideration the above-mentioned structure of the spectrum of the matrix $A_{\mathcal{C}}$ (which differs from $\tilde{A}$ only by transposition), it is 
possible to state that -1 does not belong to a spectrum of the matrix $\tilde{A}$. It guarantees that the matrix $I+\tilde{A}$ would be invertible and will allow to copy the matrix $G_{1}(s)$ as follows:

$$
\begin{aligned}
G_{1}(s) & =I+\tilde{K}^{T}(I s-\tilde{A})^{-1}(I+\tilde{A}) K_{1}, \\
K_{1} & =(I+\tilde{A})^{-1}\left(B_{1}-S \tilde{K} R_{1}\right) .
\end{aligned}
$$

Using relations (4.4), (4.5), we will obtain the following expression for $G_{1}(s)$ :

$$
G_{1}(s)=\tilde{K}^{T}(I s-\tilde{A})^{-1} K_{1}(I s+I)+I-\tilde{K}^{T} K_{1} .
$$

We will show that $I-\tilde{K}^{T} K_{1}=0$, and therefore, according to (4.1), (4.2),

$$
\Phi(s)=G_{*}(s) R_{1} G(s),
$$

where

$$
G(s)=\tilde{K}^{T}(I s-\tilde{A})^{-1} K_{1} .
$$

Really, according to (4.1), we have

$$
\Phi_{1}(-1)=G_{1 *}(-1) R_{1}\left(I-\tilde{K}^{T} K_{1}\right)=0 .
$$

However, the matrices $G_{1 *}(-1)$ and $R_{1}$ are invertible, therefore $I-\tilde{K}^{T} K_{1}=$ 0 and the outcome of the $J$-factorization of the matrix (1.3) for $R=0$ is determined by expression (4.12). Note that the matrices $G(s)$ and $G^{-1}(s)=$ $(I s+I) G_{1}^{-1}(s)$ have no poles in $\mathbb{C}_{+}$because the matrices $G_{1}(s)$ and $G_{1}^{-1}(s)$ have no poles in $\mathbb{C}_{+}$.

Thus, as shown in the problem of $J$-factorization of the matrix (1.3), in which $R=0$, it is possible to reduce to an equivalent problem with an invertible matrix $R$. The similar procedure of reduction of an initial problem can be successfully used in the general case if in such problem matrix $R$ is singular, but not equal to zero (in $[1,11]$ such problem for $Q=0$ was explicitly considered).

EXAMPLE 4.1. Let in (1.3) $C=0, R=0, A=\left[\begin{array}{ccc}0 & 1 & 0 \\ 0 & 0 & 1 \\ 5 & -1 & 5\end{array}\right], B=\left[\begin{array}{lll}0 & 0 & 1\end{array}\right]^{T}$, and $Q=h^{T} h, h=\left[\begin{array}{ll}0 & -2\end{array}\right]$. In this case,

$$
\Phi(s)=\frac{\left(s^{2}-4\right) s^{2}}{\left(s^{2}+1\right)^{2}\left(s^{2}-25\right)} .
$$

The matrix $\Phi(s)$ has zeros $(s=0)$ and poles $(s= \pm i)$ on the imaginary axis. The Hamiltonian matrix, which is corresponding to the ARE (4.8), will have zeros on the imaginary axis. According to (4.6), we have $R_{1}=1$. Using algorithms 
$[12,13,14]$ for solution ARE (4.8), we will obtain the following matrices:

$$
\begin{aligned}
& P=\left[\begin{array}{ccc}
0.000 & 0.000 & 0.000 \\
-0.000 & 4.000 & -0.000 \\
0.000 & -0.000 & 0.000
\end{array}\right], \\
& S=\left[\begin{array}{ccc}
0.0082 & 0.0408 & 0.2041 \\
0.0408 & 0.2041 & 1.0204 \\
0.2041 & 1.0204 & 5.1020
\end{array}\right] .
\end{aligned}
$$

We will further receive matrices which appear in (4.12):

$$
\begin{gathered}
\tilde{A}=\left[\begin{array}{ccc}
0 & 0.4286 & -0.2857 \\
0 & -2.8571 & -0.4286 \\
5.0000 & -15.2857 & -2.1429
\end{array}\right], \\
\tilde{K}=\left[\begin{array}{c}
0 \\
2.0000 \\
1.0000
\end{array}\right], \quad K_{1}=\left[\begin{array}{c}
-0.0000 \\
0.0000 \\
1.0000
\end{array}\right] .
\end{gathered}
$$

Using these matrices in the procedure ss2tf.m of the Matlab package, we get the following expression for $G(s)$ :

$$
G(s)=\frac{\left(s^{2}+2 s\right)}{\left(s^{3}+5 s^{2}+s+5\right)} .
$$

Obviously, zeros and poles of $G(s)$ do not lay in $\mathbb{C}_{+}$.

EXAMPLE 4.2. In the issue of Example 4.1, we will increase multiplicity of zeros and poles located on the imaginary axis:

$$
\Phi(s)=\frac{\left(s^{2}-4\right) s^{6}}{\left(s^{2}+1\right)^{4}\left(s^{2}-25\right)} .
$$

In this case,

$$
\begin{gathered}
C=0, \quad R=0, \quad A=\left[\begin{array}{ccccc}
0 & 1 & 0 & 0 & 0 \\
0 & 0 & 1 & 0 & 0 \\
0 & 0 & 0 & 1 & 0 \\
0 & 0 & 0 & 0 & 1 \\
5 & -1 & 10 & -2 & 5
\end{array}\right], \\
B=\left[\begin{array}{l}
0 \\
0 \\
0 \\
0 \\
1
\end{array}\right], \quad h=\left[\begin{array}{lllll}
0 & 0 & 0 & -2 & 1
\end{array}\right] .
\end{gathered}
$$


Having executed the procedures mentioned in Example 4.1, we will find that the matrix $P$ has all zero elements except for $P(4,4)=4$; the matrix $S$ looks as follows:

$$
S=\left[\begin{array}{lllll}
0.0000 & 0.0001 & 0.0003 & 0.0016 & 0.0082 \\
0.0001 & 0.0003 & 0.0016 & 0.0082 & 0.0408 \\
0.0003 & 0.0016 & 0.0082 & 0.0408 & 0.2041 \\
0.0016 & 0.0082 & 0.0408 & 0.2041 & 1.0204 \\
0.0082 & 0.0408 & 0.2041 & 1.0204 & 5.1020
\end{array}\right]
$$

Accordingly, we get

$$
\begin{aligned}
\tilde{A} & =\left[\begin{array}{lllcl}
0.0000 & 1.0000 & 0.0000 & -0.0229 & -0.0114 \\
0.0000 & 0.0000 & 1.0000 & -0.1143 & -0.0571 \\
0.0000 & 0.0000 & 0.0000 & 0.4286 & -0.2857 \\
0.0000 & 0.0000 & 0.0000 & -2.8571 & -0.4286 \\
5.0000 & -1.0000 & 10.0000 & -16.2857 & -2.1429
\end{array}\right], \\
\tilde{K} & =\left[\begin{array}{l}
0 \\
0 \\
0 \\
2 \\
1
\end{array}\right], \quad K_{1}=\left[\begin{array}{l}
0 \\
0 \\
0 \\
0 \\
1
\end{array}\right] .
\end{aligned}
$$

As well as is in Example 4.1, using these matrices in procedure ss2tf.m of the Matlab package, we get the following expression for $G(s)$ :

$$
G(s)=\frac{s^{4}+2 s^{3}}{s^{5}+5 s^{4}+2 s^{3}+10 s^{2}+s+5} .
$$

Obviously, zeros and poles of $G(s)$ do not lay in $\mathbb{C}_{+}$.

EXAMPLE 4.3 [1]. Let in (1.3) $A=-I, B=I, C=0, R=0$, and let the matrix $Q$ be invertible. In this case, the matrix $\Phi(s)$ has poles equal to \pm 1 , which will be cancelled with zeros of the matrices $\Psi(s), \Psi_{*}(s)$, in the process of evaluation of matrix $\Phi_{1}(s)$, according to (4.6).

We will illustrate in this example, as mentioned above, that the passage to the variable $\bar{s}$ removes the complexities, which is connected with coinciding zeros of the matrices $\Psi(s), \Psi_{*}(s)$ and poles of the matrix $\Phi(s)$. Let $\alpha=0.5$, that is, $s=0.5 \bar{s}$. In this case,

$$
\Phi(\bar{s})=4(I \bar{s}+2 I)_{*}^{-1} Q(I \bar{s}+2 I)^{-1} .
$$

In this matrix $A=-2 I, B=2 I, C=0$, and $R_{1}=0$. According to (4.6), we have

$$
B_{1}=-B=-2 I, \quad C_{1}=Q B=2 Q, \quad R_{1}=4 Q .
$$


Obviously, the stabilizing solutions of the ARE (4.8) will be $P=0, S=0$. Further, we will receive $\tilde{K}=(1 / 2) I, K_{1}=2 I$. According to (4.12),

$$
G(\bar{s})=(I \bar{s}+2 I)^{-1} .
$$

Using the variable $s$, we will receive

$$
G(s)=\frac{1}{2}(I s+I)^{-1} .
$$

Taking into consideration that $R_{1}=4 Q$, we will be convinced that the matrix $\Phi(s)$, defined by (4.11), coincides with the given matrix.

\section{Appendices}

A. Construction of the stabilizing solution of ARE. Following [13, 14], we will briefly consider the algorithm of construction of the stabilizing solution of ARE

$$
S F+F^{T} S-S G S+Q=0
$$

Solution, which will be searched below, has the following property: matrix $F-S G$ has no eigenvalues in $\mathbb{C}_{+}$. This algorithms can be interpreted as generalization of sign function method [9] of construction of the stabilizing solution of ARE, whose Hamiltonian matrix has eigenvalues on the imaginary axis. The algorithm includes the procedure of excluding stable unobservable modes, as it permits to reduce the initial problem.

So, we will assume that there is the orthogonal matrix $Z$, which transforms the matrices $F, Q$ to the following form (for algorithm of construction of matrix $Z$, see Appendix B):

$$
\bar{F}=Z F Z^{T}=\left[\begin{array}{cc}
f_{-} & * \\
0 & F_{1}
\end{array}\right], \quad \bar{Q}=Z Q Z^{T}=\left[\begin{array}{cc}
0 & 0 \\
0 & Q_{1}
\end{array}\right] .
$$

In (A.2), the sizes of the blocks $F_{1}$ and $Q_{1}$ are coinciding, the matrix $f_{-}$has no eigenvalues in $\mathbb{C}_{+}\left(\operatorname{Re}\left(\lambda\left(f_{-}\right)\right) \leq 0\right)$. The entries of the matrix $\bar{F}$, denoted by $*$, are not important. That is, the matrix $f_{-}$determines stable unobservable modes. We will transform the ARE (A.1) to the following form:

$$
\bar{S} \bar{F}+\bar{F}^{T} \bar{S}-\bar{S} \bar{G} \bar{S}+\bar{Q}=Q, \quad \bar{G}=Z G Z^{T}=\left[\begin{array}{ll}
g_{1} & g_{2} \\
g_{2}^{T} & g_{3}
\end{array}\right], \bar{S}=Z S Z^{T} .
$$

The sizes of the blocks $g_{1}, g_{2}, g_{3}$ correspond to the partitioning (A.2). We will search for the solution of the ARE (A.1) in the form

$$
\bar{S}=\left[\begin{array}{cc}
0 & 0 \\
0 & S_{1}
\end{array}\right],
$$


where the size of the block $S_{1}$ is coinciding with the size of the matrix $F_{1}$. As it follows from (A.2), (A.3), and (A.4), the matrix $S_{1}$ satisfies the ARE

$$
S_{1} F_{1}+F_{1}^{T} S_{1}-S_{1} g_{3} S_{1}+Q_{1}=0
$$

which (by assumption) has symmetric solution. This solution ensures absence of the matrix $F_{1}-g_{3} S_{1}$ in $\mathbb{C}_{+}$eigenvalues.

The Hamiltonian matrix, which corresponds to the ARE (A.5), has the form

$$
H_{1}=\left[\begin{array}{cc}
F_{1} & -g_{3} \\
-Q_{1} & -F_{1}^{\prime}
\end{array}\right] .
$$

Let the matrix $T$ transform the matrix (A.6) to the block-diagonal form

$$
H_{1}=T^{-1} \operatorname{diag}\{A, B, C\} T \text {, }
$$

where $\operatorname{Re}(\lambda(A)) \neq 0, \lambda(B)=0$, and $\operatorname{Re}(\lambda(C))=0, \lambda(C) \neq 0$. The sizes of the matrices $B$ and $C$ are equal to $2 n b \times 2 n b$ and $2 n c \times 2 n c$, respectively. Let $\pm i \omega, \ldots, \pm i \omega_{m}$ be the various eigenvalues of the matrix $C$. As $f(\lambda)$ is denoted by the polynomial, degree of which is equal to $2 m$ and the zeros of which are coinciding with the above-mentioned eigenvalues.

It is supposed that there are the integers $k b, k c \geq 1$ such that the ranks of the matrices $B^{k b}$ and $(f(C))^{k c}$ are equal to $n b$ and $n c$, respectively.

At this suppositions, the unknown solution of the ARE (A.5) is determined by the following relation:

$$
\left(\operatorname{diag}\left\{h_{1}, h_{2}, h_{3}\right\}\right) T\left[\begin{array}{c}
I \\
S_{1}
\end{array}\right]=0,
$$

where $h_{1}=I+\operatorname{sign}(A), h_{2}=B^{k b}$, and $h_{3}=(f(C))^{k c}$.

Finally, we find, according to (A.4), the solution of the ARE (A.1)

$$
S=Z^{T}\left[\begin{array}{cc}
0 & 0 \\
0 & S_{1}
\end{array}\right] Z
$$

B. Elimination of unobserved modes. It is possible to use orthogonal transformations, which are transforming system's matrices to this or that canonical form (see, e.g., [17]), for constructing the matrix $Z$, which appears in (A.2). So, let the matrices $F, Q \in \mathbb{R}^{n \times n}$ be given. Let the rank of a matrix $Q$ be equal to $r$ $(0<r \leq n)$. We will represent the matrix $Q$ as

$$
Q=c^{T} \Lambda c
$$


where $\Lambda \in \mathbb{R}^{r \times r}$ is a diagonal matrix, $c \in \mathbb{R}^{r \times n}$. It is supposed that the pair of matrices $(F, c)$ is not completely observable, that is, the observability matrix

$$
\left[\begin{array}{c}
c \\
c F \\
\vdots \\
c F^{n-1}
\end{array}\right]
$$

has a rank $p<n$. Using algorithm of orthogonal transformations (it is possible to use procedure obsvf.m of the Matlab package), we will transform the pair of matrices $(F, c)$ to canonical observability form. Namely, we will construct an orthogonal matrix $U$ such that

$$
U F U^{T}=\left[\begin{array}{cc}
f_{11} & f_{12} \\
0 & f_{22}
\end{array}\right], \quad c U^{T}=\left[\begin{array}{ll}
0 & c_{1}
\end{array}\right] .
$$

By orthogonal transformation, with a matrix $V$, we will transform the square matrix $f_{11}$ (which determines unobserved modes) to the block triangular form

$$
V f_{11} V^{T}=\left[\begin{array}{cc}
f_{-} & f_{0} \\
0 & f_{+}
\end{array}\right], \quad \operatorname{Re} \lambda\left(f_{-}\right) \leq 0, \quad \operatorname{Re} \lambda\left(f_{+}\right)>0, \quad f_{-} \in \mathbb{R}^{q \times q} .
$$

It is possible to accept, as the matrix $Z$, the following orthogonal matrix:

$$
Z=D U, \quad D=\left[\begin{array}{ll}
V & 0 \\
0 & I
\end{array}\right]
$$

(the size of the unity block $I$ in the matrix $D$ is coinciding with the size of the matrix $f_{22}$ ). Actually,

$$
Z F Z^{T}=\left[\begin{array}{cc}
f_{-} & * \\
0 & F_{1}
\end{array}\right], \quad Z Q Z^{T}=\left[\begin{array}{cc}
0 & 0 \\
0 & c_{1}^{T} \Lambda c_{1}
\end{array}\right],
$$

where $F_{1} \in \mathbb{R}^{(n-q) \times(n-q)}$. It is possible to accept the lower diagonal block of the matrix $Z Q Z^{T}$, as the matrix $Q_{1} \in \mathbb{R}^{(n-q) \times(n-q)}$, which appears in (A.2).

ACKNOWLEDGment. The author would like to thank the reviewers for their valuable suggestions.

\section{REFERENCES}

[1] F. A. Aliev and V. B. Larin, Optimization of Linear Control Systems. Analytical Methods and Computational Algorithms, Stability and Control: Theory, Methods and Applications, vol. 8, Gordon and Breach Science Publishers, Amsterdam, 1998.

[2] B. D. O. Anderson, An algebraic solution to the spectral factorization problem, IEEE Trans. Automatic Control 12 (1967), no. 4, 410-414. 
[3] B. D. O. Anderson, K. L. Hitz, and N. D. Diem, Recursive algorithm for spectral factorization, IEEE Trans. Circuits and Systems CAS-21 (1974), no. 6, 742750.

[4] D. J. Clements, Rational spectral factorization using state-space methods, Systems Control Lett. 20 (1993), no. 5, 335-343.

[5] D. J. Clements, B. D. O. Anderson, A. J. Laub, and J. B. Matson, Spectral factorization with imaginary-axis zeros, Linear Algebra Appl. 250 (1997), 225-252.

[6] M. Green, K. Glover, D. Limebeer, and J. Doyle, A J-spectral factorization approach to $H_{\infty}$ control, SIAM J. Control Optim. 28 (1990), no. 6, 1350-1371.

[7] C.-H. Guo and P. Lancaster, Analysis and modification of Newton's method for algebraic Riccati equations, Math. Comp. 67 (1998), no. 223, 1089-1105.

[8] I. Karelin, L. Lerer, and A. C. M. Ran, J-symmetric factorizations and algebraic Riccati equations, Recent Advances in Operator Theory (Groningen, 1998), Oper. Theory Adv. Appl., vol. 124, Birkhäuser, Basel, 2001, pp. 319-360.

[9] Ch. S. Kenney and A. J. Laub, The matrix sign function, IEEE Trans. Automat. Control 40 (1995), no. 8, 1330-1348.

[10] H. Kwakernaak and M. Šebek, Polynomial J-spectral factorization, IEEE Trans. Automat. Control 39 (1994), no. 2, 315-328.

[11] V. B. Larin, Factorizing about the imaginary axis matrices which do not satisfy the coercitivity, J. Automat. Inform. Sci. 28 (1996), no. 5-6, 116-130.

[12] _ Algorithm for solving algebraic Riccati equation which has singular Hamiltonian matrix, Systems Control Lett. 36 (1999), no. 3, 231-239.

[13]___ Generalized matrix sign function algorithm for algebraic Riccati equation, Proceedings of the 14th IFAC Triennial World Congress (Beijing, 1999). Riccati Equation and Algorithms, 1999, pp. 141-146.

[14]_, Generalized matrix sign function algorithm for algebraic Riccati equation, Stab. Control Theory Appl. 3 (2000), no. 2, 126-137.

[15] _ J J-factorization of rational matrices that have zeros and poles on the imaginary axis, Problemy Upravlen. Inform. (2001), no. 1, 23-33 (Russian).

[16] A. J. Laub, A Schur method for solving algebraic Riccati equations, IEEE Trans. Automat. Control 24 (1979), no. 6, 913-921.

[17] P. Hr. Petkov, N. D. Christov, and M. M. Konstantinov, Computational Methods for Linear Control Systems, Prentice-Hall, New York, 1991.

Vladimir B. Larin: Institute of Mechanics, National Academy of Sciences of Ukraine, 3 Nesterov Street, 03057 Kiev, Ukraine

E-mail address: 1arin@uaac.freenet.kiev.ua 


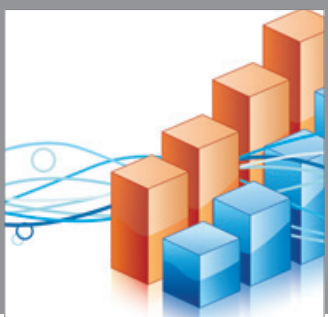

Advances in

Operations Research

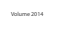

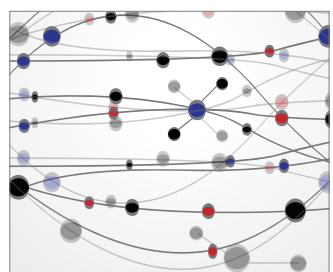

\section{The Scientific} World Journal
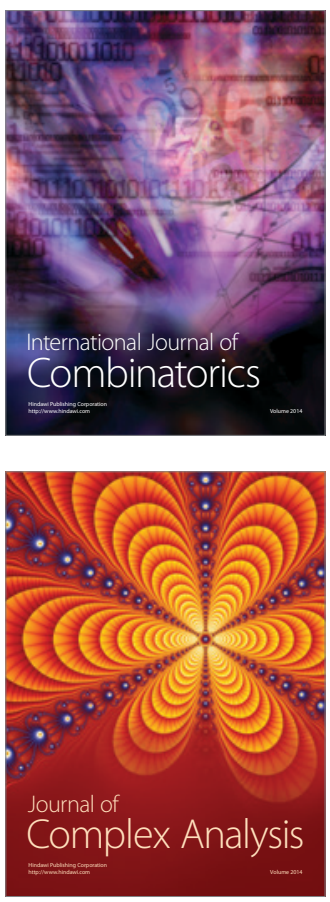

International Journal of

Mathematics and

Mathematical

Sciences
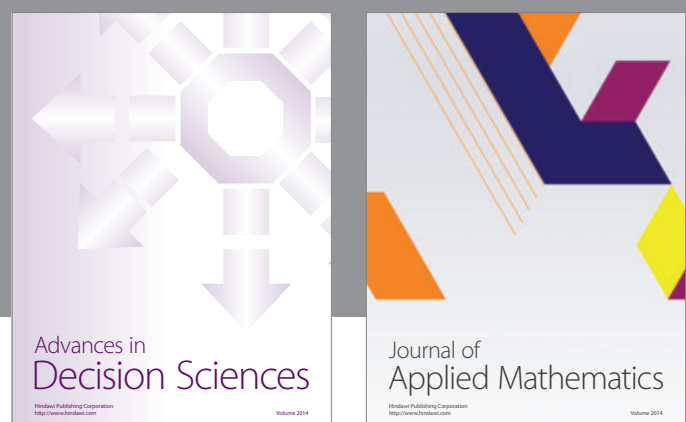

Journal of

Applied Mathematics
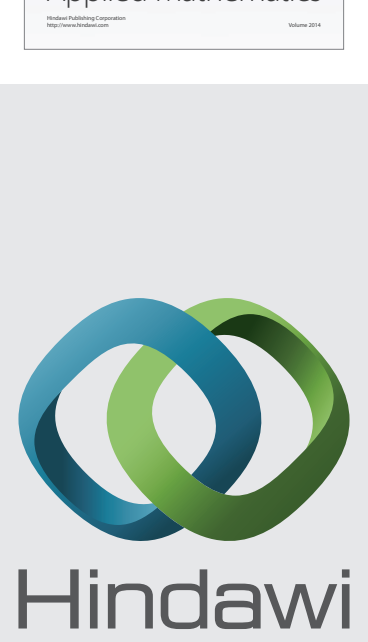

Submit your manuscripts at http://www.hindawi.com
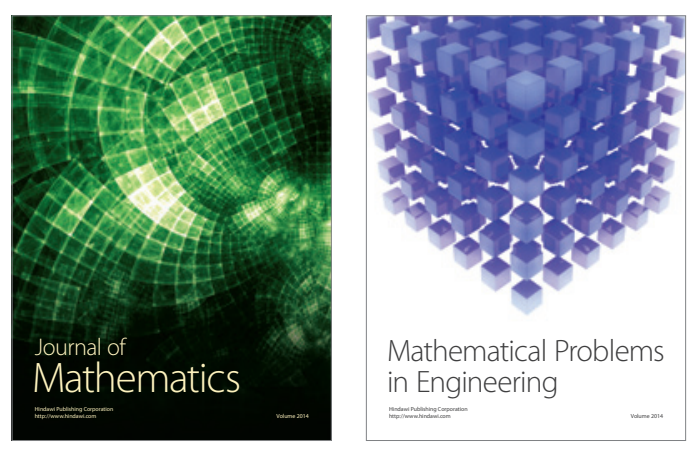

Mathematical Problems in Engineering
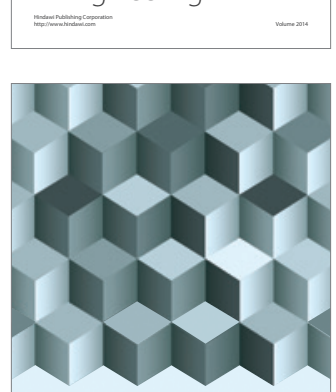

Journal of

Function Spaces
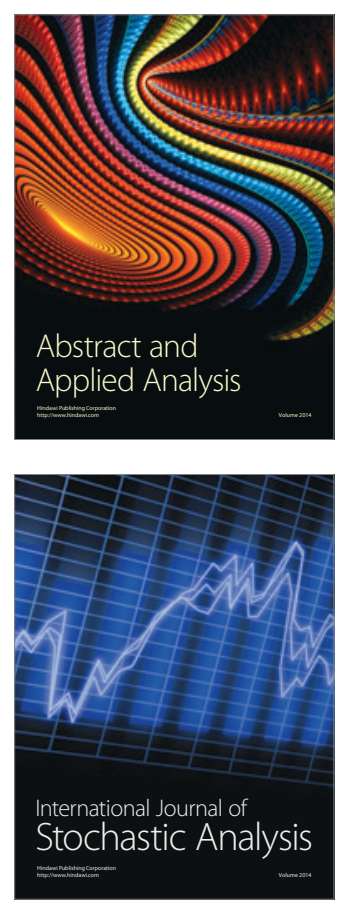

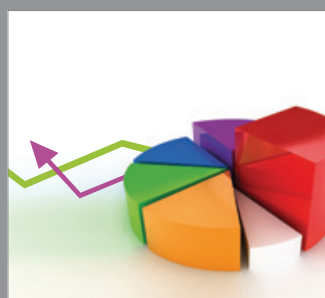

ournal of

Probability and Statistics

Promensencen
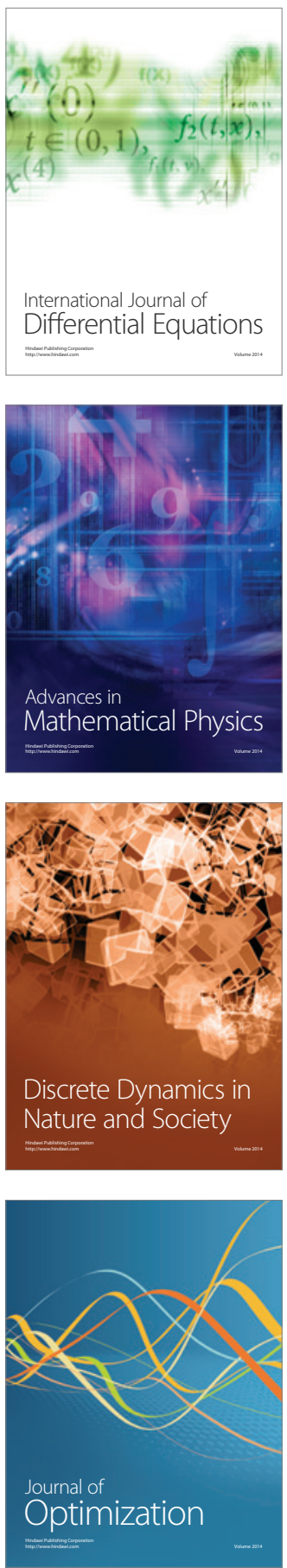